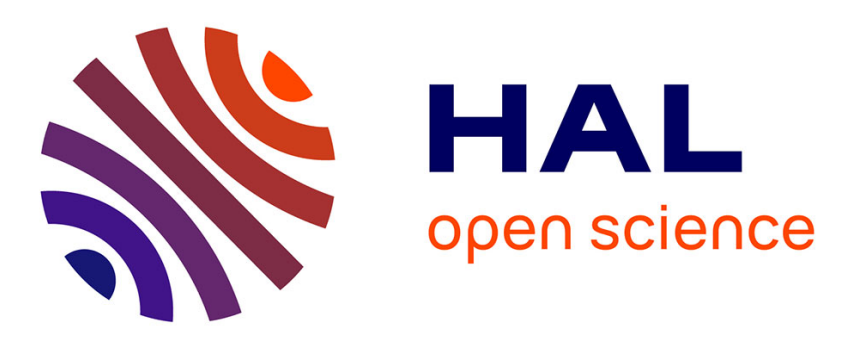

\title{
A Mobility Model For UAV Ad hoc Network
}

Ons Bouachir, Alinoé Abrassart, Fabien Garcia, Nicolas Larrieu

\section{To cite this version:}

Ons Bouachir, Alinoé Abrassart, Fabien Garcia, Nicolas Larrieu. A Mobility Model For UAV Ad hoc Network. ICUAS 2014, International Conference on Unmanned Aircraft Systems, May 2014, Orlando, United States. pp 383-388. hal-00998651

\section{HAL Id: hal-00998651 https://hal-enac.archives-ouvertes.fr/hal-00998651}

Submitted on 2 Jun 2014

HAL is a multi-disciplinary open access archive for the deposit and dissemination of scientific research documents, whether they are published or not. The documents may come from teaching and research institutions in France or abroad, or from public or private research centers.
L'archive ouverte pluridisciplinaire HAL, est destinée au dépôt et à la diffusion de documents scientifiques de niveau recherche, publiés ou non, émanant des établissements d'enseignement et de recherche français ou étrangers, des laboratoires publics ou privés. 


\title{
A Mobility Model For UAV Ad hoc Network
}

\author{
Ouns Bouachir, Alinoe Abrassart, Fabien Garcia, Nicolas Larrieu \\ The French University for Civil Aviation (ENAC), Toulouse, France \\ \{ons.bouachir, fabien.garcia, nicolas.larrieu\}@enac.fr \\ alinoe.abrassart@free.fr
}

\begin{abstract}
With the technological advances, there is an increasing attention on micro-UAVs in the military area as well as in the civilian domain. They are used as swarm (several UAVs) forming a UAS (Unmanned Aircraft System) since they are relatively cheap and offer better performance than one aircraft.

The UAVs, in a UAS, have to exchange information with each other and with the control station in order to create a clear vision of the swarm situation and the task performance. This exchange is made possible by the application of an ad hoc network between UAVs which is a challenging issue because of the node mobility, the network topology change, and the operation communication requirements in term of quality of service (delay, throughput or loss rate for instance).

This paper presents a realistic mobility model designed for UAV ad hoc networks. since evaluating the performances of ad hoc protocols is an important step in order to predict possible problems that can affect the system in the real environment. This mobility model behavior is compared to the well-known mobility model behavior Random-Way Point. It is also compared to real movements traces using several metrics.
\end{abstract}

Index Terms-Mobility models, Ad hoc networks, UAV, Paparazzi system.

\section{INTRODUCTION}

Nowadays, utilization of UAVs has become extremely popular thanks to automation and sensor technology advances that encourages many researchers to study the use of several collaborative UAVs in order to reduce the human intervention as much as possible ([1], [2], [3], [4] and [5]). UAVs have to exchange information with each other (inside the swarm) and with the control station (outside the swarm) in order to meet the needs of applications such as «cooperative task allocation» [4], «path planning» [5] or «communication relaying» [6].

The Mobile Ad hoc Network (MANET) [7] is a solution to deliver this information to its destination over long ranges via one or multiple relays [8] (Figure 1). In fact, MANET is multi-hop wireless network: each node in the network act as a mobile wireless terminal as well as a router to forward information to its neighbors. Thus, all nodes in the network are connected without requiring a pre-existing infrastructure (such as satellites or central stations with several antennas) which makes MANET a cost-effective technology. Moreover, nodes in an ad hoc network have the possibility to move freely, they move toward and away from other nodes which creates new links and breaks others. Therefore, MANET necessitates specific communication protocols in order to be an autonomous system that can faces dynamic, random, sometimes rapidly changing multi-hop topology due to UAVs mobility. Routing protocols are an example of these protocols, they are the responsible for discovering the network topology and for establishing routes to each destination. A route is the list of intermediate nodes (relays) traversed by each message in order to achieve its destination.

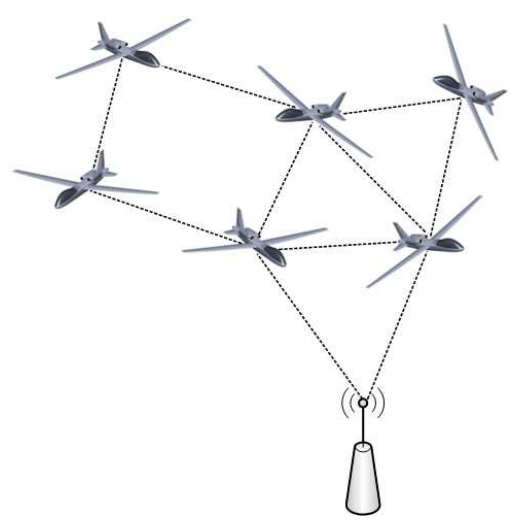

Fig. 1: UAV Ad hoc Network

The evaluation of an ad hoc network in order to predict possible problems that can affect the communication system, is achievable by resorting either to experimentation networks, called test-bed, or to simulation, using network simulators. Test-beds enable the experimentation of protocols and their application in a real environment. In general, several drawbacks hinder the realization of a test-bed [9], mainly, the inherent lack of flexibility (i.e. scenarios repetition) and the difficulty of managing and deploying network monitoring mechanisms. The monitoring should have minimal impact on the normal operation of the network; it has to use minimal computing, storage, and bandwidth resources. These difficulties can impede the network scalability [8].

On the contrary, simulations allow an easier way to monitor the network since it consists in a synthetic environment which models experiments. Protocols and experimentations are described as reproducible scenarios in files that are easily modifiable. Consequently, simulation is the most widely used solution because it offers a viable alternative, although it idealizes situations since it models them.

Mobility models are one of these features of the simulation environment. They define trajectories and speed variations 
of the mobile nodes and represents their positions, which generate network topology changes and then communication perturbations since new links will be created and others will be broken. Therefore, the mobility model plays an important role in the evaluation of the ad hoc network performance [10].

This paper presents a realistic mobility model designed for UAV ad hoc networks based on the Paparazzi UAV movements, presented in section III-A, then it compares it to another mobility model and UAV real traces as a validation mean using several metrics selected from the literature.

It is organized as follow: the first section presents some related work. The second section introduces Paparazzi system and the context of this study, then it presents the realistic mobility model designed for UAVs. After that, The validation part is presented in the third section, then the conclusion and the future work end this paper.

\section{RELATED WORK}

Mobility models are indispensable for ad hoc network research in order to create a realistic simulation environment. In fact, in [10], authors showed how the performance of an ad hoc protocol can vary significantly with different mobility models. They compared the impact of the most common mobility models on a well-known ad hoc routing protocol, Dynamic Source Routing Protocol (DSR) [11]. Results showed that DSR performance is greatly affected by mobility model changes. Another study in [12], showed that the mobility model affect the network performance. Therefore, the mobility model has to be the most closely matched to the expected real environment. For this reason, many researchers chose to create realistic models for MANET like in [13], [14] and [15]. However, fewer propositions are studied for UAV ad hoc networks like in [16], and in [17]. In general, The wellknown «Random-Way Point mobility model» (RWP) [10] is used in such situation.

In [16], authors presented two mobility models for reconnaissance application: «random mobility model» and «distributed pheromone repel mobility model». These mobility models are based on three actions: going straight, turn left and turn right. With the random mobility model proposed, UAVs decide on their actions according to fixed probabilities while with pheromone model, a pheromone map is used to guide UAVs. The aircraft exchange information about their scanned area, and according to what they decide, they turn left, right or go straight ahead.

In [17], the Semi-Random Circular Movement «SRCM», is presented. This mobility model is designed for the curved movement scenarios of UAVs, it is suitable for simulating UAVs turning around a specific position in order to gather some information.

In [18], authors proposed two mobility models for airborne networks based on circular trajectories. They permit changing flight altitude. The first model captures the correlation of movement among all three dimensions while in the second, z-dimensional movement (altitude) is independent from the two other dimensions.

The mobility model validation issue is not addressed in the literature. There is no common methodology to validate a new mobility model or to compare it to another model. Authors in [10] compared common mobility patterns between each other using only network performance measures like data packet delivery ratio or hop count.

Other papers develop new models and compare them to other models or to real traces. Indeed, in [16], authors compared the two proposed models only to each other using a geometric criterion: the scan coverage and a network measure: the UAV connectivity. This comparison did not show the effect of the mobility-related information exchange on the behavior of the network.

Away from the UAV networks, in [19], authors evaluated their new mobility model based on the comparison with real movement traces using two properties: the contact duration (the time interval in which two devices are in radio range) and the inter-contacts time (the frequency and the probability of being in contact with the packet receiver). The idea of comparing the new mobility model with real traces seems to be a good way to validate the behavior of the new mobility model.

The validation process we defined for our study will be described in section IV-A of this paper.

\section{PAPARAZZI MOBILITY MODEL (PPRZM)}

\section{A. Paparazzi System}

Paparazzi [20] is an open source system of hardware and software for the UAS: for fixed-wing aircraft as well as multicopters. It is composed by an automatic pilot, a ground station mission planner and monitoring software. Paparazzi is being used by a number of universities, companies and hobbyists all over the world and it has been developed at ENAC (French University for Civil Aviation).

In the context of the European project D3CoS [21] «Designing Dynamic Distributed Cooperative Human-Machine Systems» which focuses on the cooperation between different types of intelligent agents (such as UAVs, cars, boats, airplanes), the communication between several Paparazzi UAVs and the ground station over an ad hoc network is studied as an example of collaborative agents.

The communication system plays an important role in the success of such cooperative applications. Therefore, it is necessary to evaluate communication protocols which will be used. This evaluation can be realized by a simulation in realistic conditions to predict problems that can affect the network in the real environment. Thus, it is important to choose the appropriate mobility model. In this context, we present Paparazzi mobility model (PPRZM).

\section{B. Paparazzi Mobility Model (PPRZM)}

According to Paparazzi experts, Paparazzi UAVs have five possible movements: 
- Stay-At: the UAV hovers over a fixed position (Figure 2a);

- Way-Point: the UAV follows a straight path to a destination position (Figure $2 b$ );

- Eight: the aircraft trajectory has the $« 8 »$ form around two fixed position (Figure 2c);

- Scan: the UAV performs a scan of an area defined by two points along the round-trip trajectories (Figure 2d);

- Oval: a shifted round-trip between two points with a turn around once pass each point (Figure 2e).

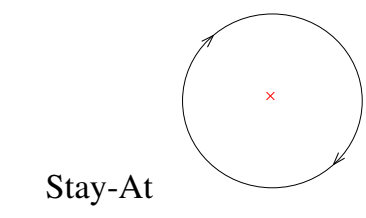

(a)

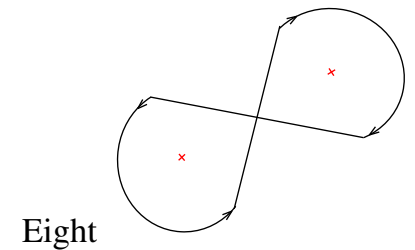

(c)

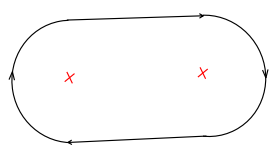

Oval

(e)

Fig. 2: Paparazzi UAV Movements

Paparazzi mobility model (PPRZM) is a stochastic mobility model that imitates Paparazzi UAV behavior based on the state machine presented in figure 3 .

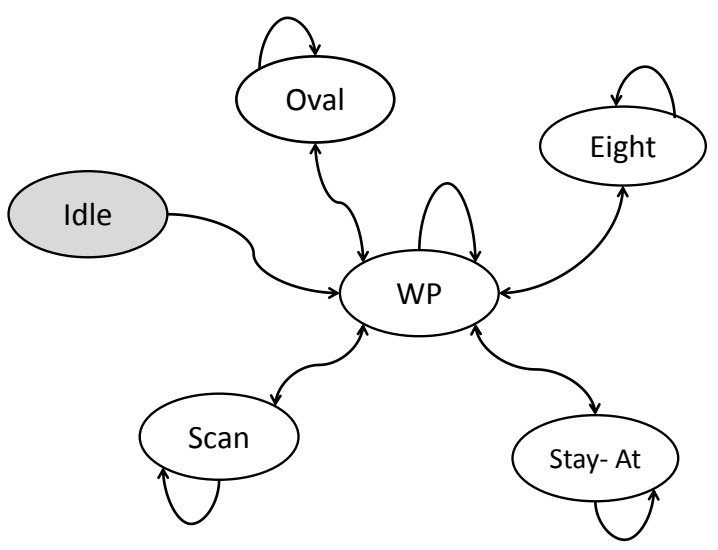

Fig. 3: PPRZM state machine
Each UAV chooses a movement type and fixes its characteristics:

- Location: the center positions for «Stay-At», «Eight» and «Oval» movements or the starting and the ending positions for «Scan» and «Way-Point» movements;

- Speed: a uniform random value between $15 \mathrm{~m} / \mathrm{s}$ and 25 $\mathrm{m} / \mathrm{s}$.

Thus, UAVs are assigned a specific position through a «Way-Point» movement, then it follows a well-defined path according to the movement chosen. Each aircraft altitude is fixed randomly at the beginning. Once reached, it remains constant till the end of the simulation.

All of these movements have different probabilities to occur. According to Paparazzi experts, «Stay-At», «Oval», and «Scan» are the movements the most produced during a mission flight. Therefore, probabilities used were fixed as follow:

- «Stay-At», «Oval», and «Scan» probabilities are equal to $30 \%$ for each movement;

- «Eight» and «Way-Point» probabilities are equal to 5\% for each movement.

PPRZM was implemented in OMNET++ [22] simulator and it is available by contacting the authors. OMNET++ is a communication network simulator. It is component-based $\mathrm{C}++$ simulation library and framework, used to evaluate communication systems and protocols.

\section{Simulations AND RESUlts}

This section presents a comparison between PPRZM, the Random Way-Point (RWP), and real Paparazzi movement traces in order to show that PPRZM has closer behavior to the real traces than RWP.

RWP is characterized by straight trajectories. Each mobile nodes selects a random destination, moves with a random speed and pauses for a «pause time» at the destination. When the pause time expires, the node chooses another random position and moves with another speed value at this location.

The Paparazzi movement traces are extracted from the Paparazzi emulator which is based on the same software used to pilot real UAVs. This emulator, also takes into account the wind conditions.

\section{A. Evaluation Means}

As presented in section II, there is no common methodology to validate a mobility model. Therefore, we chose to compare PPRZM behavior with the behavior of the wellknown mobility model RWP and with Paparazzi movement traces. This comparison is based on different measures presented in the literature. The selected metrics are classified into two classes: geometric and network related.

1) Geometric measure: geometric measures are based on the physical traces of the mobile nodes (trajectories) and their impact on the network topology. For this study, five are selected to be used: 
- Empty Cells: the simulated area is divided into cells of the same size as a grid. the cell number has to be equal to the node number. So, UAVs are uniformly distributed, if, on average, there is one aircraft per cell. This metric calculates the number of empty cells over time. It refers to the distribution uniformity of UAVs in the simulation area;

- Frequency: it is based on the same principle of dividing the simulated area into cells. It refers to the average of the number of times that a UAV crosses each cell over the visited cell number. The final value is the average over the UAV number. So, the frequency has a high value if each UAV visits limited number of cell;

- Number of Neighbor: it is the average of neighbor number per aircraft. Two nodes are neighbors if they are in range of communication. This metric refers to the possibility to create a multi-hop network. However, an important number of neighbor can affect the network performance since it increases the interference rate.

- Meeting: it is the number of created and broken links during the simulation. This measure refers to the network stability. The higher the value, the less stable the network is;

- Clustering: it is the ratio of one node neighbor number and its two hop-distance neighbor number (neighbors of neighbors). The clustering value is higher if the nodes tends to clump together in clusters.

These metrics reveal the geometric characteristics of the network, that refers to its topology, it seems to give an intuition of the difficulties that can affect the communication protocol (i.e. the possibility of creating a multi-hop network, or forming disconnected clusters, etc.) but they cannot reveal the impact of this behavior on the network. Thus, other metrics should be used to evaluate the network performance.

2) Network related measure: the network measures aims at evaluating the communication protocol performance. In this context, some metrics are selected:

- Delivery Ratio it presents the comparison between the packet received number of each UAV and the total packet sent number;

- End To End Delay: it is the delay between the first byte transmitted and the last byte received;

- Hop Count: it refers to the number of intermediate nodes used to deliver each packet to its destination.

- Rate: it is the network rate;

- RREQ Number: it is the number of routing packet sent, it refers to the difficulty that may encounter the selected routing protocol, which is AODV [23].

AODV is selected because it is one of the most popular reactive routing protocol. The reactive protocols establish routes only when needed. Therefore, they save bandwidth. Moreover, AODV implementation are readily available for simulations and real systems alike which will be an asset later in our research which will be implemented on Paparazzi UAVs.

Different importance or weight could be allocated to each measure depending on the application field of the mobility model. For this study, all selected measures have equal weight, there is no emphasis given to specific metrics. This assumption is made because we do not want to make specific utilization of PPRZM since this evaluation has a validation purpose.

\section{B. Simulation Scenario}

In order to compare PPRZM, RWP and real traces, the scenario presented in table I, is fixed;

TABLE I: Simulation scenario

\begin{tabular}{|c|c|}
\hline UAV number & 40 \\
\hline Simulation duration & $1000 \mathrm{sec}$ \\
\hline Speed & $\begin{array}{c}\text { Uniform random value } \\
\text { between }[15 \mathrm{~m} / \mathrm{s}, 25 \mathrm{~m} / \mathrm{s}]\end{array}$ \\
\hline Scan movement radius & $75 \mathrm{~m}$ \\
\hline Other circular movement radius & $80 \mathrm{~m}$ \\
\hline Simulated area & AODV \\
\hline Evaluated routing protocol & $100 \mathrm{~m}$ \\
\hline Transmission range & 802.11 \\
\hline MAC protocol & $54 \mathrm{Mbps}$ \\
\hline Channel capacity & UDP $(64 \mathrm{Bps})$ \\
\hline Traffic per UAV &
\end{tabular}

As in the real traces, the UAVs are stationary at their initial positions for the first 10 seconds. After that, they start moving until the end of the simulation.

UAVs exchange UDP messages of 64 Bytes every second over an ad hoc network using AODV routing protocol, this enables us to calculate network measures in terms of delivery ratio, end to end delay, etc.

\section{Simulation Results}

All measures of the same type (geometric and network related) are presented on a Kiviat diagram which allows to represent each metric relative to its maximum value and to facilitate the comparison between the three mobility models (PPRZM, RWP and real movement traces). For each metric, the presented values on the Kiviat are the ratio of the calculated value to the maximum value of the three mobility models. Hence, the maximum value for each measure is presented as the value 1 .

Figure 4 presents the geometric results of PPRZM, RWP and real Paparazzi traces presented on the Kiviat diagram. A first look at the chart shows a different behavior between the three simulated models which confirms that each mobility model has a unique effect on the network behavior. «Empty Cells», «Frequency» and «Meeting» measures of PPRZM are the closest to real traces ones. Therefore, PPRZM has three closer results than RWP over five since there is no emphasis given to specific metrics. 


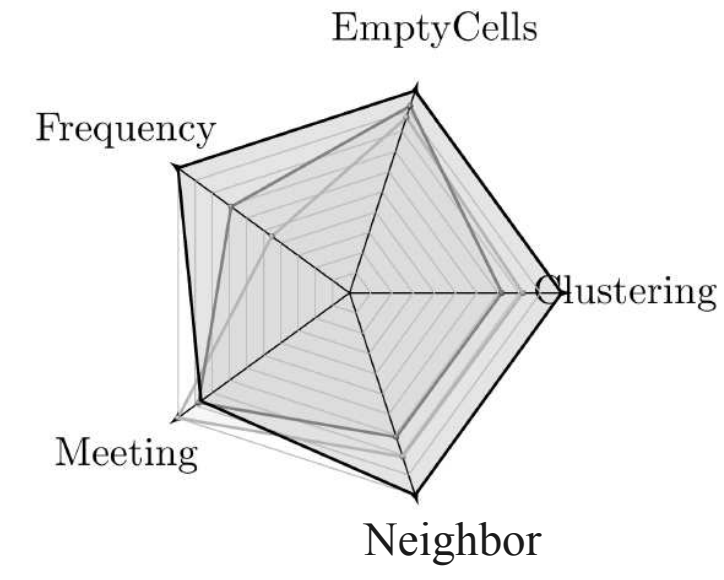

RWP

PPRZM

Real Traces

Fig. 4: Geometric comparison

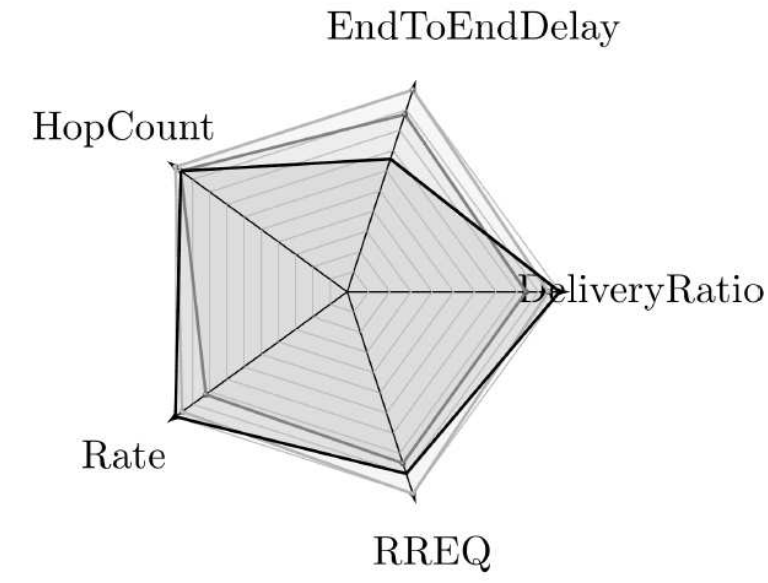

RWP

PPRZM

Real Traces

Fig. 5: Network comparison

Figure 5 presents the network performance measures of PPRZM, RWP and real Paparazzi traces. The Kiviat diagram shows, also, a difference between the behavior of the mobility models. PPRZM have three closest value to real trace ones which are: «End To End Delay», «RREQ Number» and «Hop Count».

For the second time, PPRZM has three better results than RWP over five.

For a general study, in which there is no emphasis given to specific metrics. PPRZM has the closest behavior to Paparazzi real traces.

\section{PPRZM Use Case}

PPRZM can be used to evaluate any communication protocol in the context of swarm of collaborative UAVs since it affords a realistic movement scenario. For instance, it may be used to compare several routing protocols in order to find the suitable one for UAV Ad hoc network. Moreover, PPRZM can adapt to any type of mission because it groups most UAV possible movement by changing the probability of each movement type as needed. For example, just by fixing the probability of «Scan» movement equal to 1 and all the others equal to zero, it is possible to evaluate a communication protocol in the scenario of scan mission, in which UAVs do only «Scan» movement.

\section{CONCLUSION AND FUTURE WORK}

Wireless communications are a challenging issue to provide a better performance for a UAS using several collaborating UAVs. In fact, aircraft have to exchange information about their situation and their tasks with each other as well as with the control station. In such environment, the ad hoc network is a promising solution.

In order to predict communication problems that may affect the UAS performance, ad hoc protocols have to be evaluated using a test-bed or a simulation under a realistic environment. It is important to use a realistic mobility model. In fact, the node mobility has a great effect on the network topology and the communication protocol performance.

This paper presented a realistic mobility model designed for UAV ad hoc network based on Paparazzi UAVs movements. It can be used by any researcher studying UAV networks.

First, section III introduced PPRZM (Paparazzi Mobility Model). After that, in section IV, a validation methodology was presented. It compared PPRZM to a common used mobility model, the Random Way-Point, and real UAV traces generated from Paparazzi emulator.

Since there is no common methodology to validate a mobility model, we made our comparison based on geometric and network related measures selected from the literature. For this study which has a validation purpose, all measures selected have equal weight, there is no emphasis given to specific metrics. In fact, this assumption could be modified depending on the application field of PPRZM; different weight or importance could be allocated to the selected metrics.

Results of both metric types; geometric and network performance, show that PPRZM has a closer behavior to the Paparazzi real traces than RWP.

In future work, PPRZM will be evaluated under different environment conditions and compared with other mobility models designed for UAV ad hoc network such as the models presented in section II. After that, it will be used to evaluate our QoS ad hoc architecture designed for UAV networks [24]. 


\section{ACKNOWLEDGMENT}

We would like to thank the ENAC «Paparazzi Team» for their constructive feedback and information. We also would like to thank Miss Chifa Abassi for her contribution to this work.

\section{REFERENCES}

[1] L. Wei and Z. Wei, "Path planning of uavs swarm using ant colony system," in Natural Computation, 2009. ICNC '09. Fifth International Conference on, vol. 5, pp. 288-292, 2009.

[2] S. Chaumette, R. Laplace, C. Mazel, R. Mirault, A. Dunand, Y. Lecoutre, and J.-N. Perbet, "Carus, an operational retasking application for a swarm of autonomous uavs: First return on experience," in MILITARY COMMUNICATIONS CONFERENCE, 2011 - MILCOM 2011, pp. 2003-2010, 2011.

[3] S. Waharte, N. Trigoni, and S. Julier, "Coordinated search with a swarm of uavs," in Sensor, Mesh and Ad Hoc Communications and Networks Workshops, 2009. SECON Workshops '09. 6th Annual IEEE Communications Society Conference on, pp. 1-3, 2009.

[4] Y. Jin, Y. Liao, A. A. Minai, and M. M. Polycarpou, "Balancing search and target response in cooperative unmanned aerial vehicle (uav) teams," IEEE TRANSACTIONS ON SYSTEMS, MAN AND CYBERNETICS Ü PART B: CYBERNETICS, vol. 36, no. 3, pp. 571-587, 2006.

[5] M. Polycarpou, Y. Yang, and K. Passino, "A cooperative search framework for distributed agents," in Intelligent Control, 2001. (ISIC '01). Proceedings of the 2001 IEEE International Symposium on, pp. 1-6, 2001.

[6] R. Palat, A. Annamalau, and J. Reed, "Cooperative relaying for adhoc ground networks using swarm uavs," in Military Communications Conference, 2005. MILCOM 2005. IEEE, pp. 1588-1594 Vol. 3, Oct 2005.

[7] J. M. S. Corson, "Mobile ad hoc networking (manet): Routing protocol performance issues and evaluation considerations," IETF, RFC 2501, 1999.

[8] T. X. Brown, S. Doshi, S. Jadhav, and J. Himmelstein, "Test bed for a wireless network on small uavs," pp. 20-23, 2004.

[9] K. Tan, D. Wu, A. Chan, and P. Mohapatra, "Comparing simulation tools and experimental testbeds for wireless mesh networks," in World of Wireless Mobile and Multimedia Networks (WoWMoM), 2010 IEEE International Symposium on a, pp. 1-9, June 2010.

[10] T. Camp, J. Boleng, and V. Davies, "A survey of mobility models for ad hoc network research," in Wireless Communications mobile Computing (WCMC): Special Issue On Mobile Ad hoc Networking, Research, Trends and Applications, vol. 2, pp. 483-502, 2002.

[11] D. B. Johnson, D. A. Maltz, and J. Broch, "Dsr: The dynamic source routing protocol for multi-hop wireless ad hoc networks," in In Ad Hoc Networking, edited by Charles E. Perkins, Chapter 5, pp. 139172, Addison-Wesley, 2001.

[12] A. Alshanyour and U. Baroudi, "Random and realistic mobility models impact on the performance of bypass-aodv routing protocol," in Wireless Days, 2008. WD '08. 1st IFIP, pp. 1-5, 2008.

[13] D. Gaikwad and M. Zaveri, "A novel mobility model for realistic behavior in vehicular ad hoc network," in Computer and Information Technology (CIT), 2011 IEEE 11th International Conference on, pp. 597-602, 2011.

[14] A. Kamal and J. Al-Karaki, "A new realistic mobility model for mobile ad hoc networks," in Communications, 2007. ICC '07. IEEE International Conference on, pp. 3370-3375, 2007.

[15] A. Gainaru, C. Dobre, and V. Cristea, "A realistic mobility model based on social networks for the simulation of vanets," in Vehicular Technology Conference, 2009. VTC Spring 2009. IEEE 69th, pp. 1-5, 2009.

[16] E. Kuiper and S. Nadjm-tehrani, "Mobility models for uav group reconnaissance applications," in in Proceedings of International Conference on Wireless and Mobile Communications. IEEE Computer Society, IEEE, 2006.

[17] W. Wanga, X. Guana, B. Wangb, and Y. Wangc, "A novel mobility model based on semi-random circular movement in mobile ad hoc networks," Information Sciences, vol. Volume 180, Issue 3, pp. 399_ 413, 2010.
[18] J. Xie, Y. Wan, K. Namuduri, and J. Fu, S.and Kim, "A comprehensive modeling framework for airborne mobility," in The Americain Institute of Aeronautics and Astronautics (AIAA) Conference, Boston, August 19-22, 2013.

[19] M. C. Musolesi M., "A community based mobility model for ad hoc network research," in REALMAN 'O6 Proceedings of the 2nd international workshop on Multi-hop ad hoc networks: from theory to reality, pp. 31 - 38, 2006.

[20] "Paparazzi." http://paparazzi.enac.fr/, 2014.

[21] "D3cos." http://www.d3cos.eu/, 2014.

[22] “Omnet++." http://www.omnetpp.org/, 2014.

[23] C. Perkins and E. Royer, "Ad-hoc on-demand distance vector routing," in Mobile Computing Systems and Applications, 1999. Proceedings. WMCSA '99. Second IEEE Workshop on, pp. 90-100, 1999.

[24] O. Bouachir, F. Garcia, N. Larrieu, and T. Gayraud, "Ad hoc network qos architecture for cooperative unmanned aerial vehicles (uavs)," in Wireless Days (WD), 2013 IFIP, Nov 2013. 